\title{
The Surgical Teams' Perception of the Effects of a Routine Intraoperative Pause
}

\author{
Sofia Erestam $^{1}$ (I) $\cdot$ Eva Angenete $^{1} \cdot$ Kristoffer Derwinger $^{2}$
}

Published online: 14 July 2016

(c) The Author(s) 2016. This article is published with open access at Springerlink.com

\begin{abstract}
Background A pause routine may reduce stress and errors during surgery. The aim of this study was to explore how the team, divided into the different professional groups, perceived the implementation of a pause routine and its possible impact on safety.

Methods A pause routine was introduced at a University hospital operating theatre in Sweden in 2013. Questionnaires were distributed about 1 year later to all members of the operating theatre team. The questions included different perspectives of possible effects of the pause routine.

Results A majority were positive to scheduled pauses. The surgeons often felt refreshed and at times changed their view on both anatomy and their surgical strategy. They were also perceived by other team members as improved regarding communication. All groups felt that patient safety was promoted. There were differences by profession in perception of team communication.

Conclusions The pause routine was well perceived by the surgical team. A majority believed that scheduled and regular pauses contribute to improved patient safety and better team communication. There were also findings of differences in communication and experience of team coherence between personnel categories that could benefit from further acknowledgement and exploration.
\end{abstract}

\section{Introduction}

A surgical procedure is as a team effort requiring both focus and presence, which must be maintained during the entire procedure regardless of its length $[1,2]$. There are

Sofia Erestam

sofia.erestam@vgregion.se

1 Department of Surgery, Institute of Clinical Sciences, SSORG - Scandinavian Surgical Outcomes Research Group, Sahlgrenska Academy at University of Gothenburg, Sahlgrenska University Hospital/Östra, Paviljong 11, Journalvägen 14a, 41650 Gothenburg, Sweden

2 Department of Surgery, Institute of Clinical Sciences, Sahlgrenska Academy at University of Gothenburg, Sahlgrenska University Hospital/Östra, Diagnosvägen 11, 41650 Gothenburg, Sweden several factors that may have a negative impact on the team's focus during surgery, including communication failures, environmental factors, disturbances by other personnel and technical problems, all of which may increase stress [3-9]. Stress may impair the surgical performance both at a technical and a cognitive level [10]. Acute stress has been recognized as detrimental for teamwork and may thus affect the teamwork in the operating theatre and indirectly the patient safety [11, 12]. For longer procedures, it is plausible that both physical and mental strain could contribute to stress and gradually lead to fatigue. Therefore, longer operations may benefit from an extra focus on how to handle stress and fatigue to improve patient safety.

There has been an increased interest in later years in the surgeons' non-technical skills, including leadership and communication $[13,14]$. Stress handling is also of importance 
for the surgeon. Acknowledging and coping with fatigue and stress are important. Fatigue has been recognized as a risk to patient safety through effects on cognitive performance, motor skills, communication and social skills [3, 13, 15]. It has been suggested that individual team members can increase their awareness of their own responses to stress and how to counteract this response $[10,11,16]$. Stress can be reduced by improvements of the operating theatre environment such as noise reduction [17-19], improved communication [14, 20], team building and training [21].

Intraoperative pauses also reduce stress, as described by Engelmann et al., where a strict pause discipline significantly reduced both the stress levels and the number of errors without prolonging the operating time [22]. The pause must be initiated before fatigue is evident, and this requires the setting of pause standards. The concept of scheduled pauses has many origins. It has been described from mountain expeditions where the Sherpa tradition of pacing and pause helped them reach their destinations faster than westerners going on until tired before resting. The principle is also applied by many armed forces. The aim of taking shorter pauses is to minimize the accumulation of fatigue and ameliorate ergonomics.

As an initiative to improve patient safety and surgical teamwork, a pause routine was introduced at the unit for colorectal surgery in a University Hospital in 2013. As the operation is seen as a team effort, all staff members were included in the implementation and evaluation of this routine.

The aim of this study was to explore how the team, divided into different professional categories, perceived the pause routine itself and its' implementation. The secondary aim was also to evaluate whether the different team members perceived that the pause had effects on the operation, the surgeon and team communication.

\section{Materials and methods}

\section{Setting}

The study was conducted at the Department of Surgery and the Department of Anaesthesiology at the Sahlgrenska University Hospital/Östra in Sweden. The Department of Surgery at Sahlgrenska University Hospital is a tertiary referral centre for colorectal disease, and subsequently advanced surgical procedures within both colorectal cancer and inflammatory bowel disease are performed. These procedures are often lengthy; the operating time is about 4-6 h or longer.

In 2013 a pause routine was introduced for colorectal surgical procedures and consisted of pauses every other hour, with $2 \mathrm{dl}$ of liquid refreshment and a short mental break of 1-2 min, and after every $4 \mathrm{~h}$ a longer pause with a snack or quick lunch. In addition, pauses were encouraged after the resolution of a major adverse event or when in doubt of continued surgical strategy. The anaesthetist nurses, scrub nurses and the circulating nurses were all informed that they should ask the surgeons every $2 \mathrm{~h}$ if they were ready for a short pause. The requirement for taking a pause with a short snack i.e. leaving the operating theatre was a fully stable patient. The normal operating theatre staffing at this institution was two surgeons, one scrub nurse, one anaesthetist nurse and one circulating nurse, and all but the operating surgeon remained in the operating theatre during the pause. The anaesthesiologists are responsible for several operations at a time and are not normally present in the operating theatre during the procedure and were thus not included in this study.

Prior this routine, all pauses were at the individual's initiative.

The surgical department also includes emergency surgery, upper gastrointestinal and abdominal wall surgery, but with shorter operating times, and these procedures were therefore not directly involved in this routine.

\section{Study design}

Questionnaires were handed out to all personnel categories of the operating theatre team involved in the intraoperative pause routine. The first question in the questionnaire offered personnel a chance to answer: "I am rarely active in this type of surgical procedures where pauses are current and therefore choose to not respond to the survey."

There were 16 surgeons, 19 scrub nurses, 34 anaesthetist nurses and 28 circulating nurses involved in, at least partly, the colorectal surgical team. The questions were constructed with four different response options: yes always; yes sometimes; no; and I don't know. Most questions were similar for all personnel categories, however a few questions differed. One question regarding whether the understanding of the anatomy was affected was only included in the questionnaires to the surgeons and scrub nurses as it was considered impossible for the rest of the personnel to elaborate on this subject not being close to the surgical field. Furthermore, the questions "do you experience that proposals to take a pause are received in a positive manner?", and a question about the communication with the surgeons were not addressed to the surgeons.

There was a last open-ended question giving participants the possibility of expressing their views on the subject and providing ideas of improvement.

The questionnaires were handed out after an informative meeting regarding the study at two staff meetings. After the meetings, the questionnaires were placed in the staffs' pigeonholes at the hospital. The questionnaires could be answered under full anonymity. Operation times were 
Fig. 1 Flowchart on distributed questionnaires. Note that the surgeons were all consultants at the colorectal surgery section of the department of surgery and thus involved in major surgery. The nurses of the department of anaesthesiology and operation also work towards other surgical departments with other types of surgery

attained from data registries for major surgical procedures, such as rectal cancer, both before (2011) and after (2014) the introduction of the pause routine.

\section{Statistical analysis}

The IBM SPSS Statistics 22 software package was used for analysis. Descriptive statistics were used for analysis of the questionnaires. Independent sample $t$ test was used to assess differences in operation time.

\section{Results}

The questionnaire response rate divided by professional categories is shown in Fig. 1. Many, and foremost surgeons and scrub nurses, indicated that they believed that pauses improved the team's collaboration (Fig. 2). Correspondingly, $93 \%$ of scrub nurses perceived it easier to communicate with surgeons after pauses, but this perception was less common among the anaesthetist nurses (61\% (11/ $18)$ ) and circulating nurses $(72 \%(13 / 18))$.

The surgeons stated that they often remembered to take a pause; however, the nurses' view was that the surgeons needed to be reminded. The scrub nurse was most frequent in reminding/suggesting pauses followed by the circulating nurse. Two scrub nurses had experienced negative feedback on suggestions of a pause, but a majority of the nurses (92\%) only received positive comments. Two surgeons had a routine of planning pauses in advance, however several $(67 \%)$ did occasionally. A majority of both

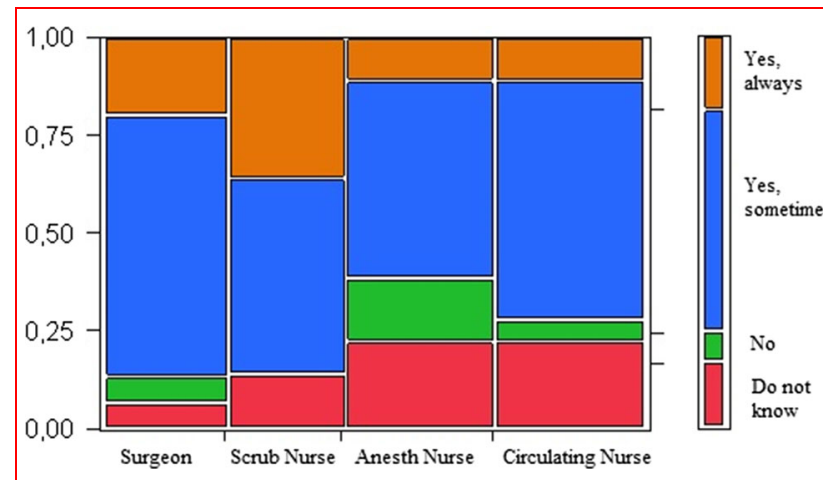

Fig. 2 Team Work. Answers in percentages by professional category to the question "Do you perceive that the team work is better after a pause?"

surgeons and the rest of the staff found the surgeons to be refreshed after a pause (Fig. 3).

Seven $(47 \%)$ surgeons said that a pause had made them change their view of the surgical anatomy, which was confirmed by $36 \%$ of the scrub nurses. $60 \%$ of the surgeons correspondingly indicated that pauses had made them change their surgical strategy. Most nurses did not know whether the surgical strategy was changed or not (Fig. 4). Three fourths of the surgeons and scrub nurses assessed that pauses had made the surgeons handle problems in a better way.

A minority $(11 \%)$ believed that pauses increased the time in the operating theatre, but a majority of the nurses marked the alternatives of "do not know" or "no change". This differed somewhat from the surgeons, where several 


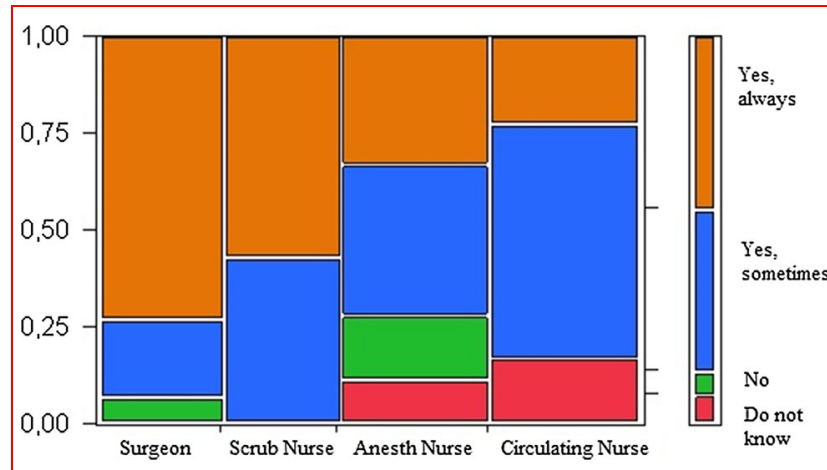

Fig. 3 Alert after pause. Answers in percentages by professional category to the question "Do you perceive that you/the surgeon is more alert after a pause?"

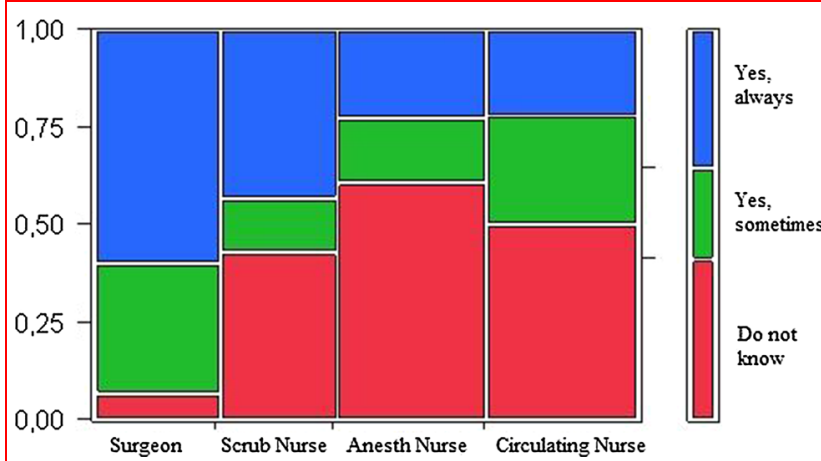

Fig. 4 The surgical strategy. Answers in percentages by professional category to the question to surgeons "Has a pause made you change surgical strategy" and to staff "Do you perceive that taking of a pause has made surgeons change surgical strategy?"

surgeons (40\% each) marked "no change" or decreased time.

A majority ( $82 \%)$ of the staff suggested that the surgeons should take pauses more often. Surgeons (93\%) and scrub nurses $(79 \%)$ experienced that the pause increased patient safety. This was less true for the anaesthetist nurses $(67 \%)$ and circulating nurses (39\%). No one answered that they believed that the patient safety decreased. Most nurses believed that the safety issue was ascertained during pauses, even when the surgeons temporarily left the operating theatre.

Notable among the written comments were remarks like "you clearly see the increasing fatigue over time with poor concentration and high irritation", "you see that the surgeons get new energy, which increase patient safety" and "I would prefer extra door openings to have alert surgeons if I was the patient". There was no significant change in operative times from before $(m=310$, SD 98.67, CI $-47.8 ; 11.7)$ to after $(m=328, \mathrm{SD} 94.38, \mathrm{CI}-47.7 ; 11.7$, $p=0.233)$ introduction of the pause routine.

\section{Discussion}

The findings in this study indicate that surgeons appreciate scheduled pauses and that it affects their performance. Engelmann et al. have previously shown interesting results in a randomized study on laparoscopic surgery with clear advantages in taking pauses [22]. The described pause schedule was every half hour, while another study on paediatric surgery by the same group had 25-min intervals [23]. They also indicated that acceptance of the pause was of importance for its effect. During the implementation of our routine, we made an effort to include the team and gain acceptance. The choice of a $2 \mathrm{~h}$ limit was an arbitrary balance between the mentioned studies above and the surgical assessment that operations lasting less than $2 \mathrm{~h}$ could be safely performed without a pause.

The findings support that the surgeons felt refreshed even after a shorter pause. This was also confirmed by the assessment of the scrub nurses. However, it is interesting that there is a difference in the perception of effect on the surgeon between the scrub nurses and the anaesthetist nurses, and the circulating nurses. A similar pattern was also seen in questions regarding team collaboration. A plausible hypothesis could be the difference in communication between different sub-groups in the team [3]. Dividing the team into two groups, those who scrubbed in work more closely together and the anaesthetist and circulating nurses who are physically a little more distant. This is however worrying as the work in the operating theatre is dependent on a team effort where all members need to be involved closely. It is possible that the surgeons as leaders in the operating theatre need to acknowledge the active participation of all team members. Reduction of practical obstacles in the operating theatre environment such as noise and background sounds could also facilitate the communication and the sense of a team effort. [13, 18].

The finding that surgeons need reminders also concurs with studies of how surgeons perceive their own skills and performance [23]. Surgeons often decline being affected by stress and fatigue during surgery $[13,24]$. We believe that the surgeons are a part of the team and need the support of the other members to keep track of time in relation to surgical progression and awareness of increased fatigue. Also, the surgeon should appreciate the team's feedback on those issues as well as of a possible notification of intraoperative fatigue. Moreover, we suggest the importance of setting good examples for younger surgeons regarding nontechnical skills including communication. The thought and environment for setting up the pause might be of more value for fatigue awareness and safety thinking, including openly asking for assistance, than just the physical side. 
The different experiences described by surgeons and nurses regarding the assessment of the anatomy or changes in surgical strategy could be explained by lack of communication. This suggests that the surgeons should communicate better with all team members by continuous short updates and teaching to improve procedural understanding and keeping the entire team updated on the progress and difficulties. The results indirectly suggest that surgeons could improve non-technical skills including team leadership through better communication and plausibly also through strategically awareness shown by time and pause planning. Also, as the results indicate that changes in perception and strategy do occur, it might support a conclusion that pauses can promote patient safety.

This study has some limitations. One is the different answer frequency by the professions in the team. Anaesthesia and circulating nurses answered to a lesser degree perhaps due to the organisation in the operating theatre. They are involved to a larger extent than surgeons and scrub nurses in many other procedures without pauses and thus they may not feel as involved. Other limitations are the risk of answer skewing bias related to ones opinion of the routine and the lack of objective data on patient safety. The latter would require another type of study with a large patient material over a long period of time. Further studies could also include semi-objective parameters such as team performance through observation by using the "Observational Teamwork Assessment for Surgery-OTAS" or "The Oxford Non-Technical Skills scale-NOTECHS $[25,26]$. One possible hard data could have been operation times. Although there was no significant change, we acknowledge that there are many confounders for time aspects. However, even with long pauses, they constitute $<10 \%$ of total times for long procedures where every misstep is an issue of both additional time to resolve and a risk for the patient.

A strength of this study was that the staff answered the questionnaire anonymously to reduce the risk of bias. Another strength was that implementation was well accepted by all surgeons; thus, there was a uniform adherence to the routine.

\section{Conclusion}

The pause routine was well perceived by the surgical team. A majority believed that scheduled and regular pauses contributed to improved patient safety and better team communication. There were also findings of differences in communication and experience of team coherence between personnel categories that could benefit from further acknowledgement and exploration.
Acknowledgments Grant support for the research reported: Sahlgrenska University Hospital (the Agreement concerning research and education of doctors), ALFGBG- 426501 and ALFGBG-493341.

Open Access This article is distributed under the terms of the Creative Commons Attribution 4.0 International License (http://crea tivecommons.org/licenses/by/4.0/), which permits unrestricted use, distribution, and reproduction in any medium, provided you give appropriate credit to the original author(s) and the source, provide a link to the Creative Commons license, and indicate if changes were made.

\section{References}

1. Healey A, Undre S, Vincent C (2004) Developing observational measures of performance in surgical teams. Qual Saf Health Care 13:i33-i40

2. Makary MA, Sexton JB, Freischlag JA et al (2006) Operating room teamwork among physicians and nurses: teamwork in the eye of the Beholder. J Am Coll Surg 202:746-752

3. Kurmann A, Tschan F, Semmer NK et al (2012) Human factors in the operating room-the surgeon's view. Trends Anaesth Crit Care 2:224-227

4. Arora S, Hull L, Sevdalis N et al (2010) Factors compromising safety in surgery: stressful events in the operating room. Am J Surg 199:60-65

5. Christian CK, Gustafson ML, Roth EM et al (2006) A prospective study of patient safety in the operating room. Surgery 139:159-173

6. Hu YY, Arriaga AF, Roth EM et al (2012) Protecting patients from an unsafe system: the etiology and recovery of intraoperative deviations in care. Ann Surg 256:203-210

7. Gillespie BM, Gwinner K, Chaboyer W et al (2013) Team communications in surgery-creating a culture of safety. J Interprof Care 27:387-393

8. Rosenstein AH, O'Daniel M (2008) A survey of the impact of disruptive behaviors and communication defects on patient safety. Jt Comm J Quality Patient Saf/Jt Comm Res 34:464-471

9. Antoniadis S, Passauer-Baierl S, Baschnegger H et al (2014) Identification and interference of intraoperative distractions and interruptions in operating rooms. J Surg Res 188:21-29

10. Wetzel CM, Kneebone RL, Woloshynowych M et al (2006) The effects of stress on surgical performance. Am J Surg 191:5-10

11. Driskell JE, Salas E, Johnston J (1999) Does stress lead to a loss of team perspective? Group Dyn 3:291-302

12. Arora S, Sevdalis N, Nestel D et al (2010) The impact of stress on surgical performance: a systematic review of the literature. Surgery $147: 318-330$

13. Flin RH, O'Connor P, Crichton MD (2008) Safety at the sharp end: a guide to non-technical skills. Ashgate, Burlington, pp 69-210

14. Lingard L, Espin S, Whyte S et al (2004) Communication failures in the operating room: an observational classification of recurrent types and effects. Qual Saf Health Care 13:330-334

15. Wetzel CM, Black SA, Hanna GB et al (2010) The effects of stress and coping on surgical performance during simulations. Ann Surg 251:171-176

16. Hull L, Arora S, Kassab E et al (2011) Assessment of stress and teamwork in the operating room: an exploratory study. Am J Surg 201:24-30

17. Kurmann A, Peter M, Tschan F et al (2011) Adverse effect of noise in the operating theatre on surgical-site infection. Br J Surg 98:1021-1025 
18. Wong SW, Smith R, Crowe P (2010) Optimizing the operating theatre environment. ANZ J Surg 80:917-924

19. Shapiro RA, Berland T (1972) Noise in the Operating Room. N Engl J Med 287:1236-1238

20. Weldon SM, Korkiakangas T, Bezemer J et al (2013) Communication in the operating theatre. Br J Surg 100:1677-1688

21. Entin EE, Serfaty D (1999) Adaptive team coordination. Hum Factors 41:312-325

22. Engelmann C, Schneider M, Kirschbaum C et al (2011) Effects of intraoperative breaks on mental and somatic operator fatigue: a randomized clinical trial. Surg Endosc 25:1245-1250

23. Engelmann C, Schneider M, Grote G et al (2012) Work breaks during minimally invasive surgery in children: patient benefits and surgeon's perceptions. Eur J Pediatr Surg 22:439-444
24. Sexton JB, Thomas EJ, Helmreich RL (2000) Error, stress, and teamwork in medicine and aviation: cross sectional surveys. BMJ 320:745-749

25. Hull L, Arora S, Kassab E et al (2011) Observational teamwork assessment for surgery: content validation and tool refinement. J Am Coll Surg 212(2):234-243

26. Mishra A, Catchpole K, McCulloch P (2009) The Oxford NOTECHS system: reliability and validity of a tool for measuring teamwork behaviour in the operating theatre. Qual Saf Health Care 18(2):104-108 